Supporting Information

\title{
Magnetically Recyclable Graphene Oxide Demulsifier Adapting Wide pH Conditions on Detachment of Oil in the Crude Oil-in-Water Emulsion
}

\author{
Haiyan $\mathrm{Xu}^{\mathrm{a}, \mathrm{b}^{*}}$, Jinqing Wang ${ }^{\mathrm{c}}$, Xiaohan Yang ${ }^{\mathrm{a}, \mathrm{b}}$, and Liping Ning ${ }^{\mathrm{a}, \mathrm{b}^{*}}$ \\ ${ }^{a}$ College of Forestry, Sichuan Agricultural University, Chengdu 611130, China. \\ ${ }^{\mathrm{b}}$ Wood Industry and Furniture Engineering Key Laboratory of Sichuan Provincial \\ Department of Education, Chengdu 611130, China. \\ ${ }^{c}$ State Key Laboratory of Solid Lubrication, Lanzhou Institute of Chemical Physics, \\ Chinese Academy of Sciences, Lanzhou 730000, China. \\ Corresponding authors, \\ E-mail: hyxu@sicau.edu.cn (H.Y.Xu); 1374515621@qq.com (L.P.Ning)
}




\section{Preparation of MR-GO}

The preparation of ferroferric oxide nanoparticles $\left(\mathrm{Fe}_{3} \mathrm{O}_{4} \mathrm{NPs}\right)$ was carried out in the basis of our previous work ${ }^{19}, 29$. The $\mathrm{Fe}_{3} \mathrm{O}_{4} @ \mathrm{SiO}_{2}$-APTES was prepared by a modified Stöber method ${ }^{30}$. The silica $\left(\mathrm{SiO}_{2}\right)$ shell was modified with organic silane coupling agent (APTES) to render a surface of amine groups. Finally, the GO reacted with the amine groups on the amino-functionalized $\mathrm{Fe}_{3} \mathrm{O}_{4}$ particles and ethylenediamine to form amino-rich magnetic GO. The detailed preparation of MR-GO was depicted as follows:

Firstly, the $\mathrm{Fe}_{3} \mathrm{O}_{4} \mathrm{NPs}$ were coated by a layer of $\mathrm{SiO}_{2}$. Typically, $0.2 \mathrm{~g}$ of $\mathrm{Fe}_{3} \mathrm{O}_{4}$ NPs was dispersed in a mixture of $160 \mathrm{~mL}$ of ethanol and $40 \mathrm{~mL}$ of water by ultrasonication for $30 \mathrm{~min}$. Then $0.6 \mathrm{~mL}$ of TEOS and $0.6 \mathrm{~mL}$ of APTES were added into the above mixture under continuously mechanical stirring, followed by the adjuction of $6 \mathrm{~mL}$ of ammonia solution. The chemical reaction was carried out at $40^{\circ} \mathrm{C}$ for $6 \mathrm{~h}$ under an atmosphere of nitrogen. The product of $\mathrm{Fe}_{3} \mathrm{O}_{4} @ \mathrm{SiO}_{2}$-APTES was collected by an external magnet and rinsed with water and ethanol, respectively.

Then, GO was synthesized from natural graphite powder by a modified Hummers method ${ }^{31,26}$. In detail, graphite (12 g) was put into a concentrated $\mathrm{H}_{2} \mathrm{SO}_{4}$ solution (50 $\mathrm{mL}), \mathrm{K}_{2} \mathrm{~S}_{2} \mathrm{O}_{6}(10 \mathrm{~g})$, and $\mathrm{P}_{2} \mathrm{O}_{5}(10 \mathrm{~g})$ and kept at $80^{\circ} \mathrm{C}$ for $8 \mathrm{~h}$. The mixture was then washed with deionized water to remove the residual acid and dried under ambient condition overnight. Next, the pretreated graphite (5g) was put into cold $98 \% \mathrm{H}_{2} \mathrm{SO}_{4}$ (200 mL), following with addition of the $\mathrm{KMnO}_{4}(25 \mathrm{~g})$ under stirring at $0{ }^{\circ} \mathrm{C}$. The asobtained mixture was stirred at $35^{\circ} \mathrm{C}$ for $4 \mathrm{~h}$, and then diluted with $250 \mathrm{~mL}$ of deionized 
water in an ice bath to keep the temperature below $50{ }^{\circ} \mathrm{C}$. After adjunction of the 250 $\mathrm{mL}$ of deionized water, the mixture was stirred for $2 \mathrm{~h}$, and then $750 \mathrm{~mL}$ deionized water was added. The mixture was treated with $50 \% \mathrm{H}_{2} \mathrm{O}_{2}$ till the color of the solution changed into brilliant yellow. The mixture was then centrifuged and washed with $10 \%$ hydrochloric acid aqueous solution (2L) till the $\mathrm{pH}$ of the solution became neutral. The resulting solid was dispersed in water by ultrasonication for 90 min to obtain GO dispersion $(0.5 \mathrm{wt} \%)$. The obtained brown dispersion was centrifugated at $4000 \mathrm{r} / \mathrm{min}$ for 10 min to remove aggregates and purified by dialysis for 1 week to remove the remained impurities. Finally, the product was obtained by vacuum freeze-drying.

Finally, the prepared GO was totally dispersed in ethylenediamine, amounts of $\mathrm{Fe}_{3} \mathrm{O}_{4} @ \mathrm{SiO}_{2}$-APTES were then added into the above mixtures and the mixture solution was heated under reflux at $120^{\circ} \mathrm{C}$ for $48 \mathrm{~h}$. The product of MR-GO was collected by magnet and washed several times by ethanol and deionized water. The MR-GO was then dried under vacuum at $60{ }^{\circ} \mathrm{C}$ for $48 \mathrm{~h}$.

\section{Characterizations}

Transmission electron microscopy (TEM, Tecnai G2 TF20)was to ovserve the morphological structures of samples. Fourier transform infrared (FT-IR) spectrometer (Nicolet, Nexus-870), X-ray photoelectron spectroscopy (XPS, PHI-5702, Physical Electronics, USA) and Raman spectroscopy (Renishaw inVia, $633 \mathrm{~nm}$ line of an Ar ion laser as an excitation source) were used to analyze the chemical structures of samples. The X-ray diffraction (XRD) (Panalytical, X' pert PRO) was carried out to investigate the phase structure of the as-prepared samples. Micromeritics ASAP 2020 V4 surface 
area $\&$ pore size analyzer was applied to test porous characteristics of the samples based on nitrogen adsorption and desorption isotherms (BET isotherms). Vibrating sample magnetometer (VSM, model Lake Shore, new 7304 series) was used to test magnetic properties of samples at room temperature. Zeta potentiometer (Brookhaven, USA) was employed to evaluate zeta potentials of of various samples at different $\mathrm{pH}$ levels.

\section{Stability of crude $\mathrm{O} / \mathrm{W}$ emulsion}

The stability of crude $\mathrm{O} / \mathrm{W}$ emulsion in the in the $\mathrm{pH}$ of $2.0-10.0$ has been measured, as shown in Fig. S1. It is obvious that the oil absorbance has little decrease at various $\mathrm{pH}$ levels of emulsion (blue line). Moreover, in comparison with the original crude $\mathrm{O} / \mathrm{W}$ emulsion, the oil absorbance has little reduction after settling for $48 \mathrm{~h}$, demonstrating the crude $\mathrm{O} / \mathrm{W}$ emulsion has good stability. The result suggested that the influence of $\mathrm{pH}$ levels on emulsifier can be ignored.

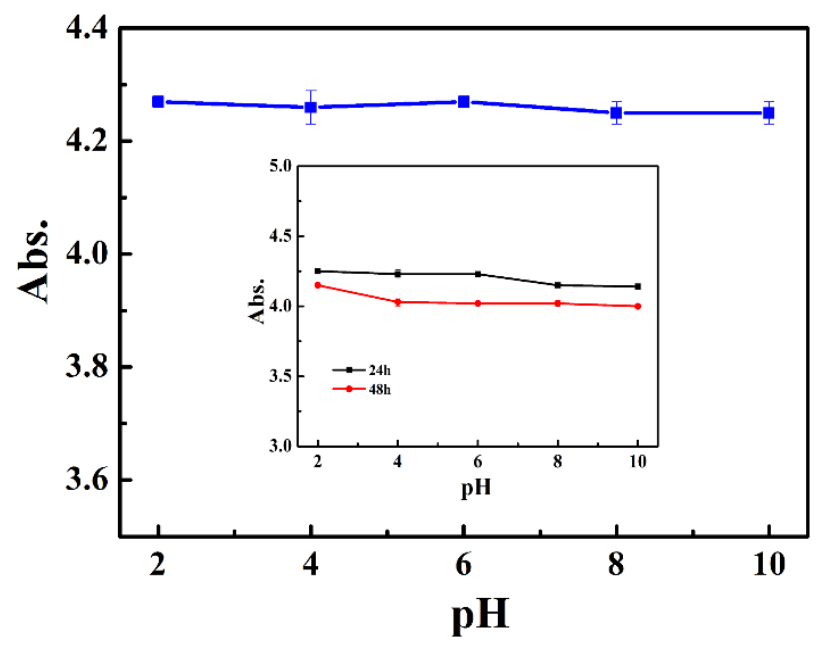

Fig. S1 Effect of the $\mathrm{pH}$ levels and settling time on stability of crude O/W emulsion

\section{The standard curve of oil content-oil absorbance}

Crude oil diluent with different concentrations were prepared via adjunction of 1.0 $\mathrm{mL}$ of crude oil into petroleum ether and then diluted to different times. Then the oil 
absorbances at various concentrations are tested at $225 \mathrm{~nm}$, as described in Figure S2.

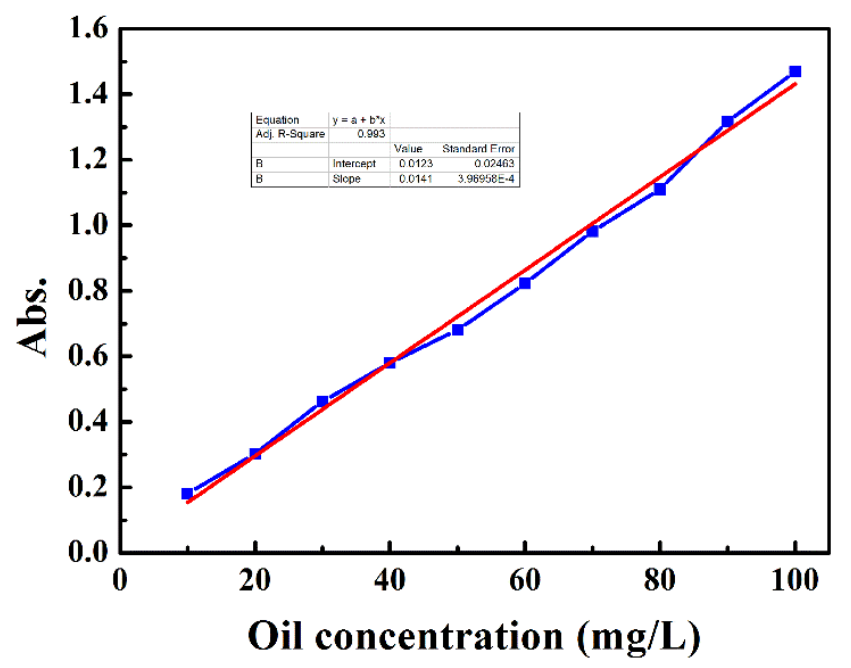

Figure S2 The standard curve of oil concentration-oil absorbance

\section{The energy-dispersive spectrum (EDS) of $\mathrm{Fe}_{3} \mathrm{O}_{4}$ and $\mathrm{Fe}_{3} \mathrm{O}_{4} @ \mathrm{SiO}_{2}-\mathrm{APTES}$}

(a)

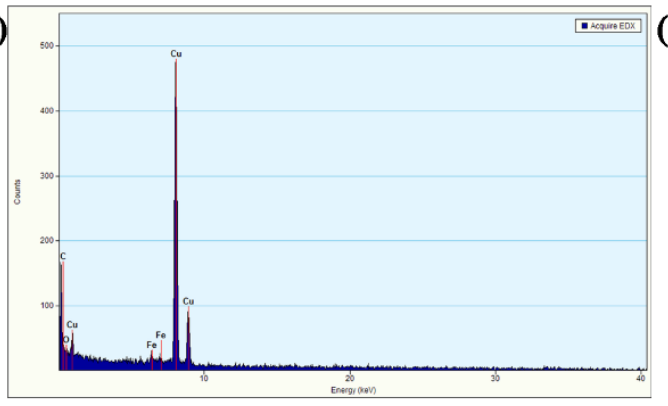

(b)

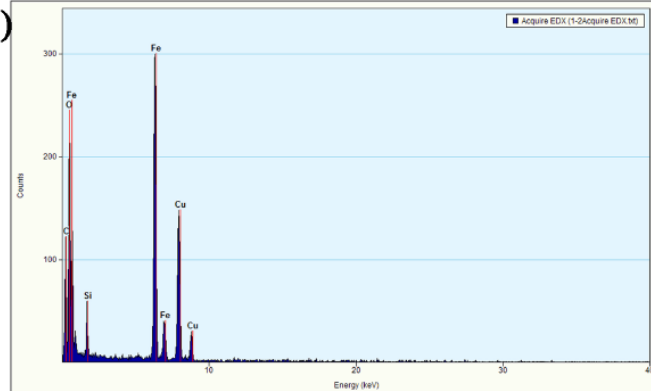

Figure $\mathrm{S} 3 \mathrm{EDS}$ of $\mathrm{Fe}_{3} \mathrm{O}_{4}(\mathrm{a})$ and $\mathrm{Fe}_{3} \mathrm{O}_{4} @ \mathrm{SiO}_{2}$-APTES (b)

The EDS of $\mathrm{Fe}_{3} \mathrm{O}_{4}$ and $\mathrm{Fe}_{3} \mathrm{O}_{4} @ \mathrm{SiO}_{2}$-APTES have been showed in Figure S3. The EDS of $\mathrm{Fe}_{3} \mathrm{O}_{4}$ presented that only $\mathrm{Fe}, \mathrm{O}$ and $\mathrm{C}$ elements are detected (a). For the $\mathrm{Fe}_{3} \mathrm{O}_{4} @ \mathrm{SiO}_{2}$-APTES, besides $\mathrm{Fe}, \mathrm{O}$ and $\mathrm{C}$ elements of $\mathrm{Fe}_{3} \mathrm{O}_{4}$, additional $\mathrm{Si}$ and $\mathrm{N}$ elements are tested in the $\mathrm{Fe}_{3} \mathrm{O}_{4} @ \mathrm{SiO}_{2}$-APTES (b), confirming the $\mathrm{SiO}_{2}$ and APTES have been coated on the $\mathrm{Fe}_{3} \mathrm{O}_{4}$ surface.

\section{The XPS spectra of various samples}

XPS measurements were employed to analyze the clearer chemical composition 
and the element information of the samples. The XPS survey spectrum of $\mathrm{Fe}_{3} \mathrm{O}_{4} @ \mathrm{SiO}_{2}-$ APTES, GO and MR-GO are displayed in Figure S4(a). The high-resolution XPS spectra of O1s and N1s for these samples are described in Figure S4(b-d). The peak at $531.4 \mathrm{eV}$ is ascribed to the oxygen atoms in carboxyl or carbonyl groups, while the peak at $532.5 \mathrm{eV}$ is assigned to epoxy, hydroxyl, or carboxyl groups, and the peak at $533.6 \mathrm{eV}$ is attributed to oxygen atoms in water and chemisorbed oxygen species ${ }^{26}$. In addition, the high resolution N1s XPS spectra of the $\mathrm{Fe}_{3} \mathrm{O}_{4} @ \mathrm{SiO}_{2}-\mathrm{APTES}$ and MR-GO are described in Figure S4 (e, f). It is found that two peaks appeared at different binding energies, indicating two chemical states of nitrogen located at $399.8 \mathrm{eV}$ and $400.7 \mathrm{eV}$ existed $^{38,39}$. Specifically, the peak at $399.8 \mathrm{eV}$ is assigned to the bond of $\mathrm{O}=\mathrm{C}-\mathrm{N}$, deriving from the dehydration condensation reaction of $-\mathrm{COOH}$ and $-\mathrm{NH}_{2}$ groups. Moreover, the peak at $400.7 \mathrm{eV}$ is due to the bond of C-C-N of APTES and ethylenediamine. The results demonstrated the APTES and ethylenediamine have been successfully grafted onto the $\mathrm{Fe}_{3} \mathrm{O}_{4} @ \mathrm{SiO}_{2}$ and $\mathrm{GO}$ surface. Compared with our previous test, the signal-to-noise ratios of N1s fine spectra for $\mathrm{Fe}_{3} \mathrm{O}_{4} @ \mathrm{SiO}_{2}$-APTES and MR-GO have been greatly improved. While they are still lower than O1s fine spectra, and the results are consistent with the reported literatures ${ }^{40,41}$. For the Fe $2 p$ spectra of the $\mathrm{Fe}_{3} \mathrm{O}_{4} @ \mathrm{SiO}_{2}$-APTES and MR-GO $(\mathrm{g}, \mathrm{h})$, two peaks are observed at different binding energies. The peak locating at $710.6 \mathrm{eV}$ is ascribed to the $\mathrm{Fe} 2 \mathrm{p}_{3 / 2}$, while another peak locating at $724.1 \mathrm{eV}$ is attributed to the $\mathrm{Fe} 2 \mathrm{p}_{1 / 2}^{19,42}$, indicating that the iron element existed in the MR-GO composite. 

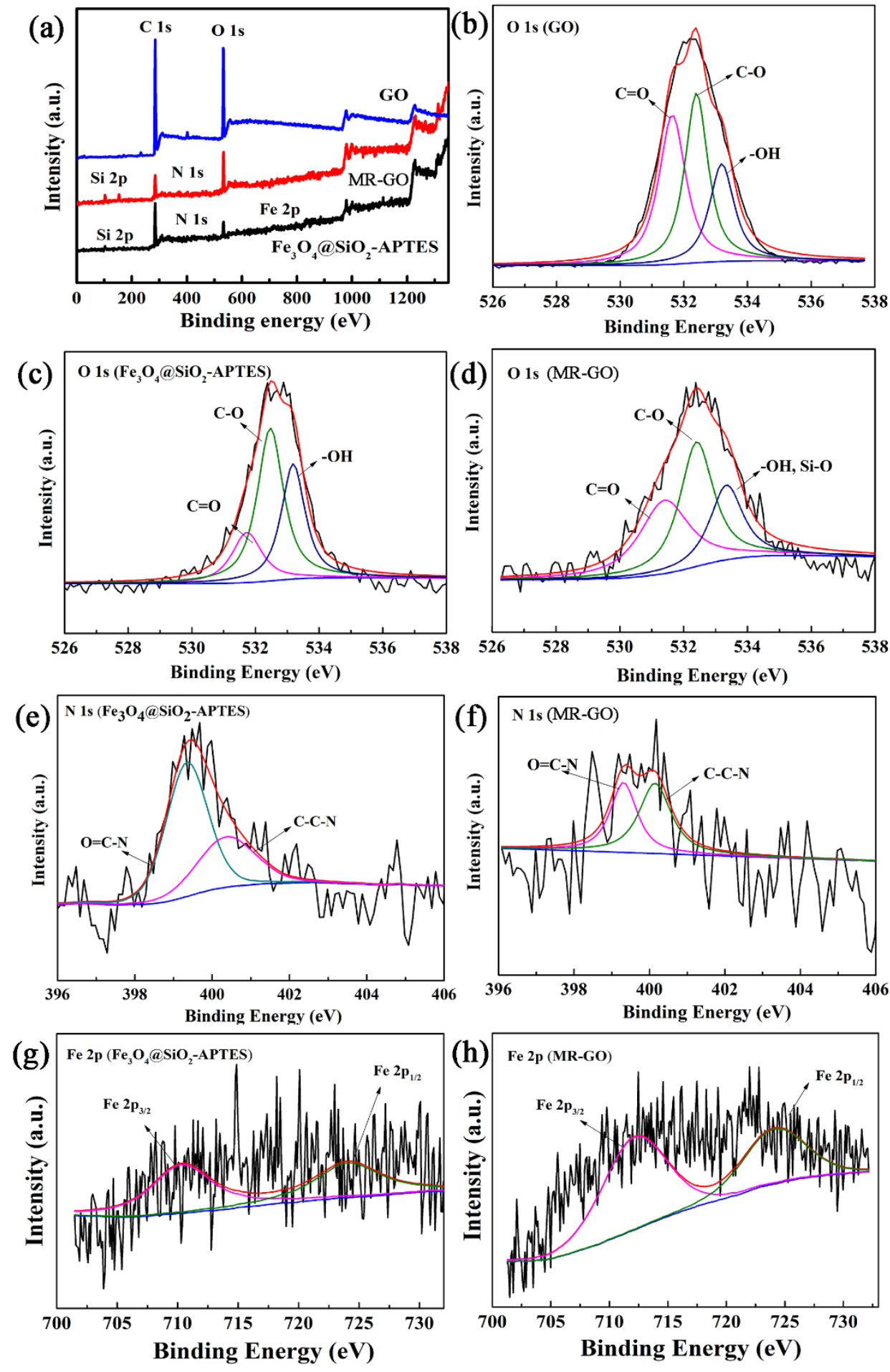

Figure S4 The XPS survey spectra results of the $\mathrm{Fe}_{3} \mathrm{O}_{4} @ \mathrm{SiO}_{2}$-APTES, GO, MR-GO;

fine spectra of O 1s (GO, $\mathrm{Fe}_{3} \mathrm{O}_{4} @ \mathrm{SiO}_{2}$-APTES, M-RGO); N 1s $\left(\mathrm{Fe}_{3} \mathrm{O}_{4} @ \mathrm{SiO}_{2}\right.$-APTES, MR-GO); Fe 2p ( $\left.\mathrm{Fe}_{3} \mathrm{O}_{4} @ \mathrm{SiO}_{2}-\mathrm{APTES}, \mathrm{MR}-\mathrm{GO}\right)$ 


\section{Zeta potential of emulsified oil droplets}

Since zeta potential of the droplets was strongly dependent on the $\mathrm{pH}$ of the continuous phase ${ }^{19, \mathrm{~S} 1-2}$, in this work, zeta potentials of crude $\mathrm{O} / \mathrm{W}$ emulsion at $\mathrm{pH}$ levels of 2.0, 4.0, 6.0, 8.0, and 10.0 were measured, respectively. As shown in Figure $5 \mathrm{a}$, the $\mathrm{O} / \mathrm{W}$ emulsion is negatively charged in the $\mathrm{pH}$ range of $2.0-10$ and the corresponding absolutely values of zeta potentials increased with the $\mathrm{pH}$ values improved. The reason was that the interfacial films composed of asphaltenes, resins, carboxylic acids and clays are commonly used to stabilize emulsified oil droplets ${ }^{\text {S3-4 }}$. For the asphaltene, it was composed of polar hydrophilic groups such as hydroxyl, carboxyl, amino, nitro and mercapto groups, as well as non-polar lipophilic groups such as alkyl groups and fused ring aromatics ${ }^{\mathrm{S5}-\mathrm{S6}}$. While for the resins, it also contained some surface active materials, including aromatic ring, long-chain carboxylic acid, phenols, and esters ${ }^{\mathrm{S} 7}$. Typically, it is proved that the hydroxyl groups and aromatic hydrocarbon could increase the negative charge of emulsified oil droplets remarkably with the $\mathrm{pH}$ increase ${ }^{\mathrm{S} 8}$. In addition, the formed hydrogen bond by hydroxyl and amino groups further improved the stability of emulsion ${ }^{\mathrm{S} 9}$. Moreover, with the increase of $\mathrm{pH}$ levels, caboxyl groups are dissociated and became negatively charged carboxylate ($\mathrm{COO}^{-}$) under alkaline conditions, and thusly improving the electrostatic repulsion of emulsified oil droplets. The results demonstrated the synergy effect of functional groups promoted the absolutely values of zeta potentials increased with the $\mathrm{pH}$ values improved. The increased absolute zeta potential values of emulsions provided a high energy barrier between emulsion droplets, thusly providing strong electrostatic 
repulsion $^{52}$.

\section{Recovery rate of the MR-GO demulsifier}

Recovery rate of the MR-GO demulsifier was also investigated according to the MR-GO dosage before demulsification and recovered by magnet after demulsification. The results are shown in Figure S5. It is found that after 1 cycles, the recovery rate of the MR-GO demulsifier was $99.0 \%$. However, it was gradually reduced and dropped to $80.3 \%$ after 6 cycles. After that, the recovery rate of the MR-GO sharply decreased. The results are consistent with Figure 7(f).

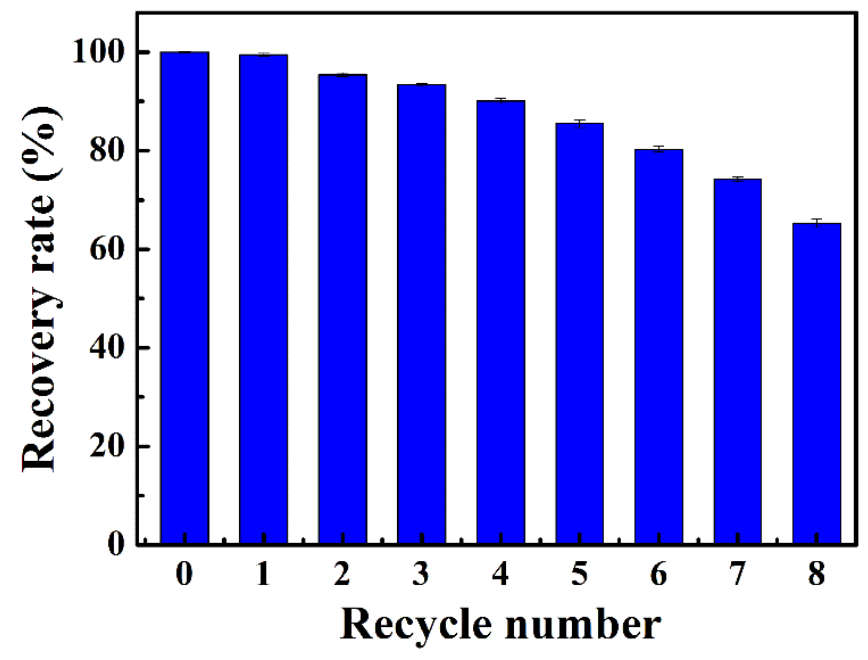

Figure S5 Recovery rate of the MR-GO demulsifier

\section{REFERENCES}

(S1) Mikulcová, V.; Bordes, R.; Minařík, A.; Kašpárková, V., Pickering Oil-in-Water Emulsions Stabilized by Carboxylated Cellulose Nanocrystals-Effect of the pH. Food Hydrocolloids 2018, 80, 60-67.

(S2) Geng, J.; Pu, J.; Zhao, Y.; Lin, B.; Bai, B.; Thomas, S. P., pH-Responsive Crude Oil-in-Water Pickering Emulsion Stabilized by Polyacrylamide Nanogels. Fuel 2019, $258,116159$. 
(S3) Razi, M.; Rahimpour, M. R.; Jahanmiri, A.; Azad, F., Effect of a Different Formulation of Demulsifiers on the Efficiency of Chemical Demulsification of Heavy Crude Oil. J. Chem. Eng. Data 2011, 56 (6), 2936-2945.

(S4) Pensini, E.; Harbottle, D.; Yang, F.; Tchoukov, P.; Li, Z.; Kailey, I.; Behles, J.; Masliyah, J.; Xu, Z., Demulsification Mechanism of Asphaltene-Stabilized Water-inOil Emulsions by a Polymeric Ethylene Oxide-Propylene Oxide Demulsifier. Energy Fuels 2014, 28 (11), 6760-6771.

(S5) Wang, Y.; Wang, B.; Wang, Q.; Di, J.; Miao, S.; Yu, J., Amino-Functionalized Porous Nanofibrous Membranes for Simultaneous Removal of Oil and Heavy-Metal Ions from Wastewater. ACS appl. Mater. Interfaces 2019, 11 (1), 1672-1679.

(S6) Rogel, E., Simulation of interactions in asphaltene aggregates. Energy Fuels 2000, $14,566-574$.

(S7) Zhang, J.; Tian, D.; Lin, M.; Yang, Z.; Dong, Z., Effect of Resins, Waxes and Asphaltenes on Water-Oil Interfacial Properties and Emulsion Stability. Colloid. Surf A 2016, 507, 1-6.

(S8) Wiacek, A.; Chibowski, E., Stability of Oil/Water (Ethanol, Lysozyme or Lysine) Emulsions. Colloid Surf B 2000, 17, 175-190.

(S9) Shi, C.; Zhang, L.; Xie, L.; Lu, X.; Liu, Q.; Mantilla, C. A.; van den Berg, F. G.; Zeng, H., Interaction Mechanism of Oil-in-Water Emulsions with Asphaltenes Determined Using Droplet Probe AFM. Langmuir 2016, 32 (10), 2302-2310. 